\title{
COMMUNICATIVE ASYMMETRY, HANDEDNESS AND LANGUAGE EXPERIENCE
}

\author{
OLGA VASILEVA \\ ovasilev@sfu.ca \\ Department of Psychology, Simon Fraser University, Vancouver, Canada
}

Lateralization of human cognitive and linguistic functioning has important implications for the evolution of our species. Various researchers suggest that a link between communicative and manual development in humans, in both ontogeny and phylogeny, led to a human-specific brain lateralization pattern for language, tool-use and possibly other cognitive domains (Corballis, 2003; Fitch $\&$ Braccini, 2013). The human-unique character of these asymmetries (namely, the fact that humans are the only animals demonstrating such strong populationlevel biases in both asymmetries), and their presumed importance for humanspecific traits, such as tool-use and language (e.g. Estalrrich \& Rosas, 2013; Chance \& Crow, 2007), have long attracted researchers' attention and became a field of studies in its own right.

However, the exact nature of handedness-language relationship, as well as its evolutionary history is far from being understood. Particularly concerning is the fact that studies conducted on human infants and primates bring inconsistent results. Some researchers (e.g. Fagard, 2013) have expressed serious concerns regarding the level of understanding of manual/communicative asymmetries relations in human ontogeny and phylogeny. Although there is evidence suggesting that language and handedness are associated, more research is required to test this hypothesis.

There are two important notions regrading such a research. First of all, it is necessary to investigate the association between language asymmetry, handedness and other cognitive parameters (Cochet, 2016). Second of all, it is necessary to conduct studies on more diverse samples. The majority of studies investigating manual and communicative asymmetries have been conducted with either human infants or non-human primates, while the number of studies addressing these aspects in the adults is quite limited. Such situation is problematic for our ability to relate the findings of research in children and primates with the research conducted on adult population. Moreover, the studies 
addressing the asymmetries in the adult population, as a rule, rely on samples with a high level of homogeneity (e.g. age, language background or handedness). However, an integrated approach to the problem of manuallinguistic asymmetries relationship demands not only comparative, but a more comprehensive developmental data, and that is, examination of manual handedness and language not only in children, but necessarily in the adults in more diverse samples. To this day, only a limited number of studies have employed such approach.

The proposed submission outlines a project attempting to apply such an integrative approach and fill in existing gaps in the research. The purpose of the project is to investigate the relationship between manual and linguistic asymmetries in relation to the participants' language experience (bilinguals/monolinguals). According to two recent meta-analyses bilinguals exhibit a more balanced asymmetry in language processing with the right hemisphere being significantly involved in language processing (Vaid \& Hull, 2006; Vaid \& Hull, 2007). Moreover, the results indicate that overall monolinguals (MG) and late bilinguals (LB) (individuals acquiring a second language after the age of six) demonstrate a more left-lateralized pattern of language processing, while the early bilinguals (EB) (individuals acquiring a second language before the age of six) are less lateralized in that respect. Consequently, since BL differ from ML in language asymmetry, and since there is a presumed connection between manual and language asymmetry in the adults (Cochet \& Vauclair, 2012), it might be expected that bilinguals also differ from monolinguals in handedness.

No previous research has addressed the question of handedness - language asymmetry in relation to bilingualism. Additionally, while many previous studies have employed indirect measures of language asymmetry (e.g. asymmetry of a communicative gesture), in the present study participants will take a dichotic listening test to determine their actual linguistic asymmetry profile (Hugdal, 2003). If language asymmetry and handedness is associated in the adults, we can expect congruent relationship between these parameters in each of the groups (EB, LB, ML) and significant differences between groups.

Finally, in order to capture the relationship between handedness and language more effectively, the study would employ additional measures allowing separating these relationships from potential co-founding parameters, such as general motor asymmetry (footedness) and cognitive factors (autism-like and schizotypy traits measured in non-clinical populations and previously associated with mixed handedness and decreased lateralization (e.g. Sommer et al., 2009). The studies are using established methodology on novel populations which allows comparing results with the existing findings in the field and, at the same time, broadening a research area of asymmetry development and its implications for the evolution of cognition and language in humans. 


\section{References}

Chance, S. A., \& Crow, T. J. (2007). Distinctively human: cerebral lateralisation and language in Homo sapiens. J Anthropol Sci, 85, 83-100.

Cochet, H. (2016). Manual asymmetries and hemispheric specialization: Insight from developmental studies. Neuropsychologia, 93, 335-341.

Cochet, H., \& Vauclair, J. (2012). Hand preferences in human adults: Noncommunicative actions versus communicative gestures. cortex, 48(8), 10171026.

Corballis, M. C. (2003). From hand to mouth: The origins of language. Princeton University Press.

Estalrrich, A., \& Rosas, A. (2013). Handedness in Neandertals from the El Sidrón (Asturias, Spain): evidence from instrumental striations with ontogenetic inferences. PLoS One, 8(5), e62797.

Fagard, J. (2013). Early development of hand preference and language lateralization: are they linked, and if so, how?. Developmental psychobiology, 55(6), 596-607.

Fitch, W., \& Braccini, S. N. (2013). Primate laterality and the biology and evolution of human handedness: a review and synthesis. Annals of the New York Academy of Sciences, 1288(1), 70-85.

Hugdahl, K. (2003). Dichotic listening: an experimental tool in clinical neuropsychology. In Experimental methods in neuropsychology (pp. 2946). Springer US.

Hull, R., \& Vaid, J. (2007). Bilingual language lateralization: A meta-analytic tale of two hemispheres. Neuropsychologia, 45(9), 1987-2008.

Somers, M., Sommer, I. E., Boks, M. P., \& Kahn, R. S. (2009). Hand-preference and population schizotypy: a meta-analysis. Schizophrenia research, 108(1), 25-32.

Vaid, J., \& Hull, R. (2006). Laterality and language experience. Laterality, 11(5), 436-464 